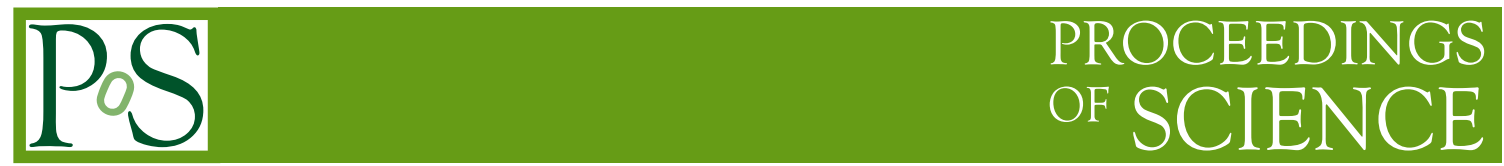

\title{
Charmonia and Exotics from BESIII: spectroscopy and production
}

\section{YUTIE LIANG*}

ON BEHALF OF THE BESIII COLLABORATION

II. Physikalisches Institut, Justus Liebig Universität Giessen, D-35392 Giessen, Germany

E-mail: yutie.liang@physik.uni-giessen.de

Recent progress on the exotic and charmonium-like states from BESIII experiment is presented, including the observation of the $1^{3} D_{2}$ candidate $X(3823)$, the observation of $X(3872)$ from $Y(4260)$ radiative decay, the study of $Y$ states, and the observation of $Z_{c}$ states.

VIII International Workshop on Charm Physics

5-9 September 2016

Bologna, Italy

\footnotetext{
* Speaker.
} 


\section{Introduction}

In quark model [1], conventional hadrons are divided into two categories, the quark-antiquark mesons and three-quark baryons. However, Quantum Chromodynamics (QCD) allows the existence of states beyond the conventional hadron, referred as exotic states, such as glueball, hybrids, multi-quark states (states consist of more than 3 quarks). The study of exotic states is important to our understanding of QCD. Despite extensive experimental searches, no unambiguous candidates for any of these exotic configurations have yet to be identified. On the other hand, a number of new particles in the charmonium mass region are observed and show different features from normal charmonium states, and might be good candidates for exotic states.

The BESIII experiment operating at the Beijing Electron Positron Collider II (BEPCII) started to take data since 2009. Since the end of 2012, the experiment has accumulated about $5 \mathrm{fb}^{-1}$ data samples with center of mass energy above $4 \mathrm{GeV}$ and yields fruitful results on the (exotic) charmonium spectroscopy. In this paper, recent progress on the exotic and charmonium(-like) states will be presented.

\section{The $\mathrm{X}$ states}

\subsection{The observation of $X(3823)$}

Recently, the Belle Collaboration reported an evidence for a narrow resonance $X(3823) \rightarrow$ $\gamma \chi_{c 1}$ in B meson decays with a significance level of $3.8 \sigma$ and mass of $(3823.1 \pm 1.8 \pm 0.7) \mathrm{MeV} / \mathrm{c}^{2}$ [2]. This resonance is considered as a good candidate for the $1^{3} D_{2}$ charmonium state.

In BESIII, a search on this $X(3823)$ resonance is performed using the process of $e^{+} e^{-} \rightarrow$ $\pi^{+} \pi^{-} \gamma \chi_{c 1}$ with $4.67 \mathrm{fb}^{-1}$ data above $4 \mathrm{GeV}$. The $X(3823)$ state is observed in the $\pi^{+} \pi^{-}$recoil mass spectra with a statistical significance of $6.2 \sigma$, as shown in the left plot of Fig. 1. The mass of the $X(3823)$ state is determined to be $(3821.7 \pm 1.3 \pm 0.7) \mathrm{MeV} / \mathrm{c}^{2}$, and the width is less than 16 $\mathrm{MeV}$ at a $90 \%$ confidence level. The energy-dependent cross section of $e^{+} e^{-} \rightarrow \pi^{+} \pi^{-} X(3823)$ is shown in Fig. 1 (Right). A fit with the $Y(4360)$ or $Y(4415)$ shape is performed, and both resonance shapes can describe the cross section line shape [3].
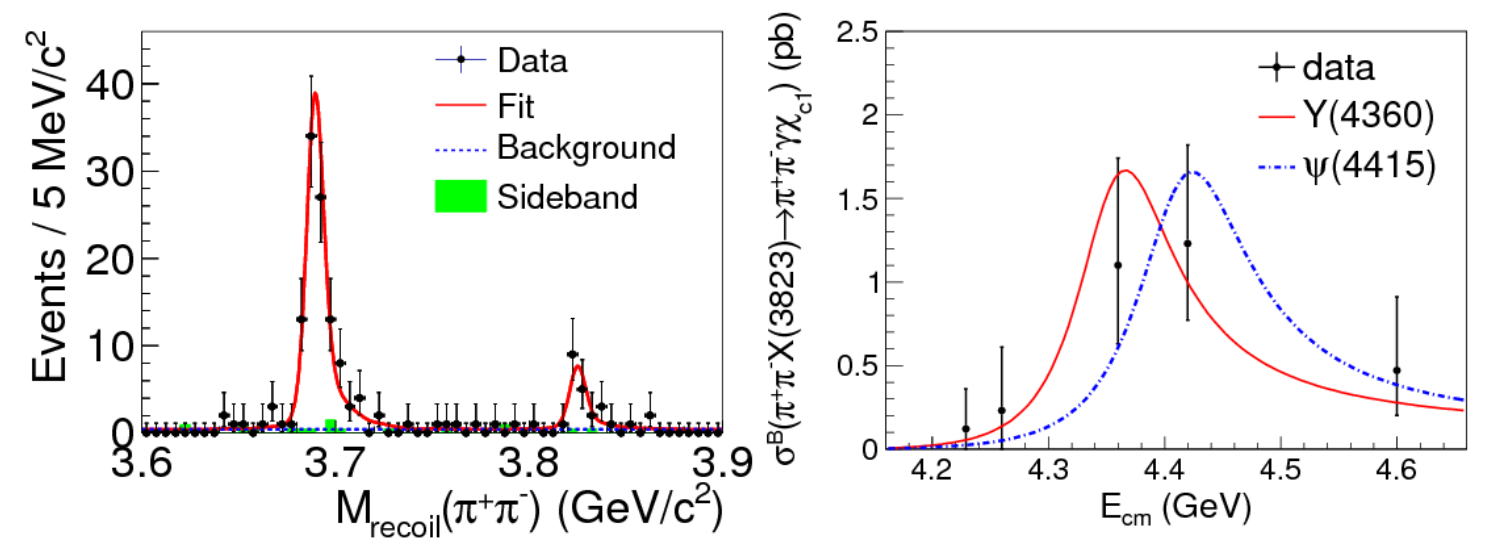

Figure 1: (Left) Fit to the $\pi^{+} \pi^{-}$recoil mass distribution of $\gamma \chi_{c 1}$ events. (Right) Fit to the energy-dependent cross section with the $Y(4360)$ (red solid curve) and the $\psi(4415)$ (blue dashed curve) line shape. 


\subsection{The observation of $e^{+} e^{-} \rightarrow \gamma X(3872)$ at BESIII}

In 2003, the $X(3872)$ state was first observed by Belle in B decays [4]. It was subsequently confirmed by several experiments. Since its mass is close to the $D D^{*}$ threshold, it has been interpreted as a candidate for a hadronic molecule or a tetraquark state.

In BESIII, using $3.3 \mathrm{fb}^{-1}$ data samples at center-of-mass energies from 4.009 to $4.420 \mathrm{GeV}$, the $X(3872)$ is observed in the process of $e^{+} e^{-} \rightarrow \gamma X$ (3872) with a significance of $6.3 \sigma$, as shown in Fig. 2 (Left). The measured mass, $(3871.9 \pm 0.7 \pm 0.2) \mathrm{MeV} / \mathrm{c}^{2}$ is consistent with the previous measurements. In addition, the energy-dependent production cross section of $e^{+} e^{-} \rightarrow \gamma X$ (3872) was measured, and shown in Fig. 2 (Right). It strongly support the existence of the radiative transition process $Y(4260) \rightarrow \gamma X(3872)$ [5].
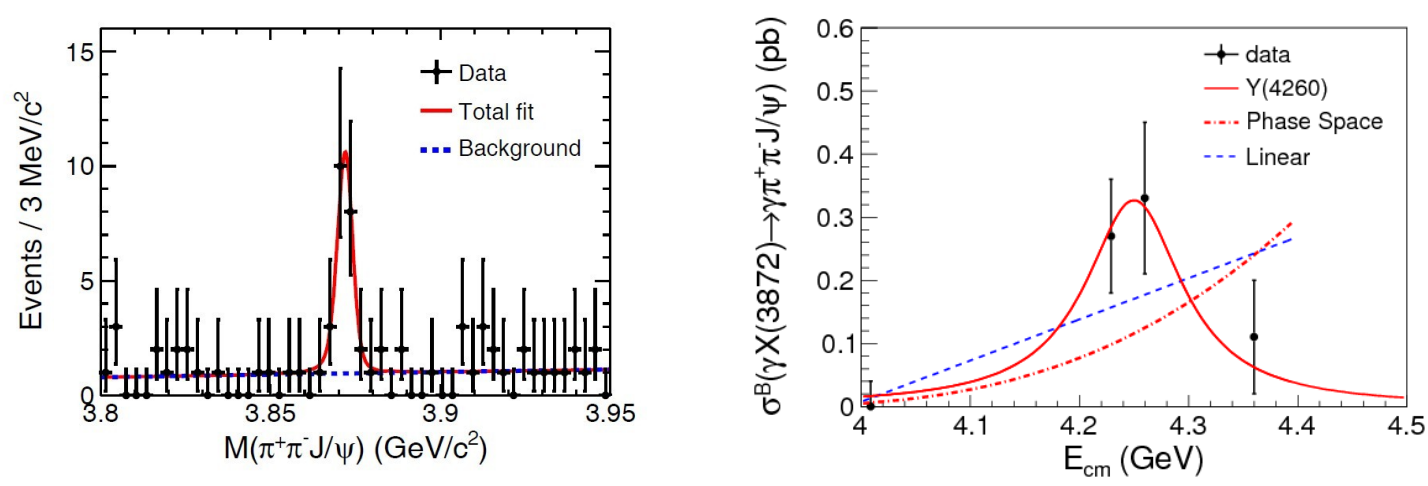

Figure 2: (Left) Fit to the invariant mass of $\pi^{+} \pi^{-} J / \psi$ for $X(3872)$ signal. (Right) Fit to the energydependent cross section with the $Y(4260)$ resonance (red solid curve) and a linear continuum (blue dashed curve) line shape and a E1-transition phase space term (red dotted-dashed curve).

\subsection{The search of $e^{+} e^{-} \rightarrow \gamma X(4140)$ at BESIII}

In 2009, an evidence for the $X(4140)$ (or $Y(4140)$ ) was reported by the CDF experiment [6]. In a subsequent analysis, $\mathrm{CDF}$ claimed the observation of this state with a statistical significance greater than $5 \sigma$. However, the existence of this state was not confirmed by the other experiments yet.

In BESIII, using data samples collected at center of mass energies of 4.23, 4.26 and 4.36 $\mathrm{GeV}$, a search of this $\mathrm{X}(4140)$ was performed through a radiative transition followed by its decay to $\phi J / \psi[7]$. No significant signal is observed and upper limits on $\sigma\left[e^{+} e^{-} \rightarrow \gamma X(4140)\right] \times$ $B(Y(4140) \rightarrow \phi J / \psi)$ at the $90 \%$ confidence level are estimated as $0.35,0.28$ and $0.33 \mathrm{pb}$ at 4.23 , 4.26 and $4.36 \mathrm{GeV}$, respectively.

\section{The Y states}

As $1^{--}$states, the Y resonances can be produced directly via $e^{+} e^{-}$annihilation. One method of studying the $\mathrm{Y}$ states is to measure the line shapes of their different channels, such as the final states of $\pi^{+} \pi^{-}$combined with a charmonium state $(J / \psi$ or $\psi(2 S))$. At B factory, the BaBar 
Collaboration and the BELLE Collaboration had measured the line shapes of these decay channels using ISR technique to lower the center of mass energy to the charmonium region [8] [9] [10] [11], which led to the observation of the Y(4260) and Y(4360). Other decay channels, such as one light meson $(\omega, \eta$, or $\eta \prime)$ combined with a charmonium state $\left(J / \psi, \chi_{c J}\right)$ are also good candidate channels for the Y studies.

Due to the rapidly reduced cross section by ISR technique, the statistics on charmonium region from B factory is limited. At BESIII, We have taken large amount of data at different energy points above $3.8 \mathrm{GeV}$ to $4.6 \mathrm{GeV}$. Many analysis concerning the $\mathrm{Y}$ study are ongoing. In this section, two analysis will be shown.

\subsection{Measurement of $e^{+} e^{-} \rightarrow \omega \chi_{c J}$}

In 2015, the processes, $e^{+} e^{-} \rightarrow \omega \chi_{c J}$, were investigated with data from 4.21 to $4.42 \mathrm{GeV}$ [12]. The final state $\omega \chi_{c 0}$ was observed around $4.23 \mathrm{GeV}$ for the first time, while $\omega \chi_{c 1,2}$ signals were not seen. By examining the $\omega \chi_{c 0}$ cross section as a function of center of mass energy, it is found inconsistent with the line shape of the $Y(4260)$ observed in $e^{+} e^{-} \rightarrow \pi^{+} \pi^{-} J / \psi$. Later, combining data samples above $4.4 \mathrm{GeV}$, the channels $e^{+} e^{-} \rightarrow \omega \chi_{c 1,2}$ are observed for the first time [13]. Fig. 3 shows the measured Born cross section (center value) for $e^{+} e^{-} \rightarrow \omega \chi_{c J}(\mathrm{~J}=0,1,2)$ as a function of the center of mass energy. The top plot is for $e^{+} e^{-} \rightarrow \omega \chi_{c 0}$, the middle plot for $e^{+} e^{-} \rightarrow \omega \chi_{c 1}$, and the bottom for $e^{+} e^{-} \rightarrow \omega \chi_{c 2}$. The triangle black points are from Ref. [12] and others are from Ref. [13]. The $\sigma\left(e^{+} e^{-} \rightarrow \omega \chi_{c 0}\right)$ is fitted with a resonance(solid curve) in the top plot. The $\sigma\left(e^{+} e^{-} \rightarrow \omega \chi_{c 2}\right)$ is fitted with the coherent sum of the $\psi(4415) \mathrm{BW}$ function and a phase-space term. The solid curve shows the fit result, the blue dashed curve is the phase-space term, which is almost the same for the two solutions. The purple dash-dotted curve is the destructive solution and the green dash-double-dotted curve is the constructive one.

The different line shapes observed for $\omega \chi_{c J}$ might indicate that the production mechanism is different, and that nearby resonances have different branching fractions to the $\omega \chi_{c J}$ decay modes. Further studies based on more data samples at a higher energy will be helpful to clarify the nature of charmonium(like) states in this region.

\subsection{Observation of $e^{+} e^{-} \rightarrow \eta / J / \psi$}

The process $e^{+} e^{-} \rightarrow \eta / J / \psi$ is investigated using data samples collected with the BESIII detector at 14 c.m. energies from 4.189 to $4.600 \mathrm{GeV}$ [14]. Significant $e^{+} e^{-} \rightarrow \eta / J / \psi$ signals are observed at 4.226 and $4.258 \mathrm{GeV}$ for the first time, and the corresponding Born cross sections are measured to be $(3.7 \pm 0.7 \pm 0.3)$ and $(3.9 \pm 0.8 \pm 0.3) \mathrm{pb}$, respectively. Assuming that the $\eta / J / \psi$ signals come from the $\psi(4160)$ decay, the cross section is fitted with a constant-width relativistic BW function, as shown by the red curve in Fig. 4. The statistical significance of the $\psi(4160)$ is 6.6 $\sigma$. The second resonance $\psi(4415)$ is added coherently in the fit, and the statistical significance of it is $1.3 \sigma$, which indicates that the contribution of $\psi(4415)$ is not significant.

\section{Observations of the $Z c$ states}

4.1 Observations of $Z_{c}(3900)$ and $Z_{c}(3885)$

In 2013, BESIII discovered a charged charmonium-like state $Z_{c}(3900)$ decaying into $\pi^{ \pm} J / \psi$ 


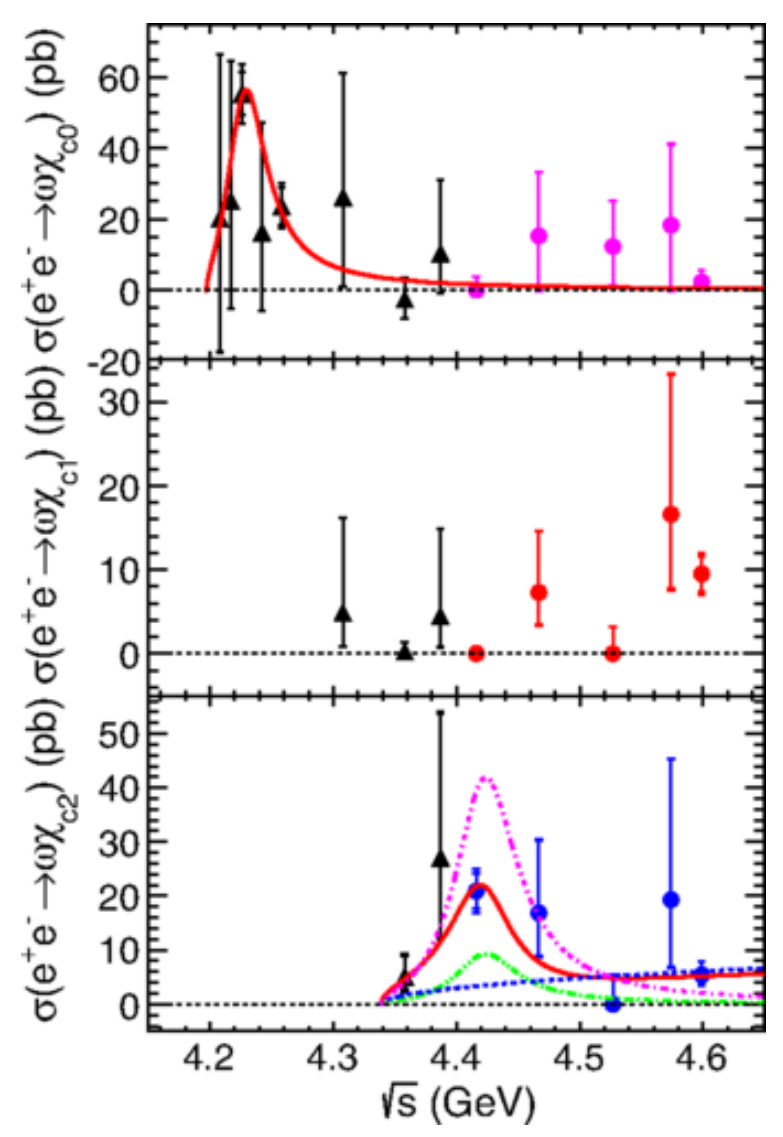

Figure 3: Measured Born cross section (center value) for $e^{+} e^{-} \rightarrow \omega \chi_{c J}(\mathrm{~J}=0,1,2)$ as a function of the center of mass energy.

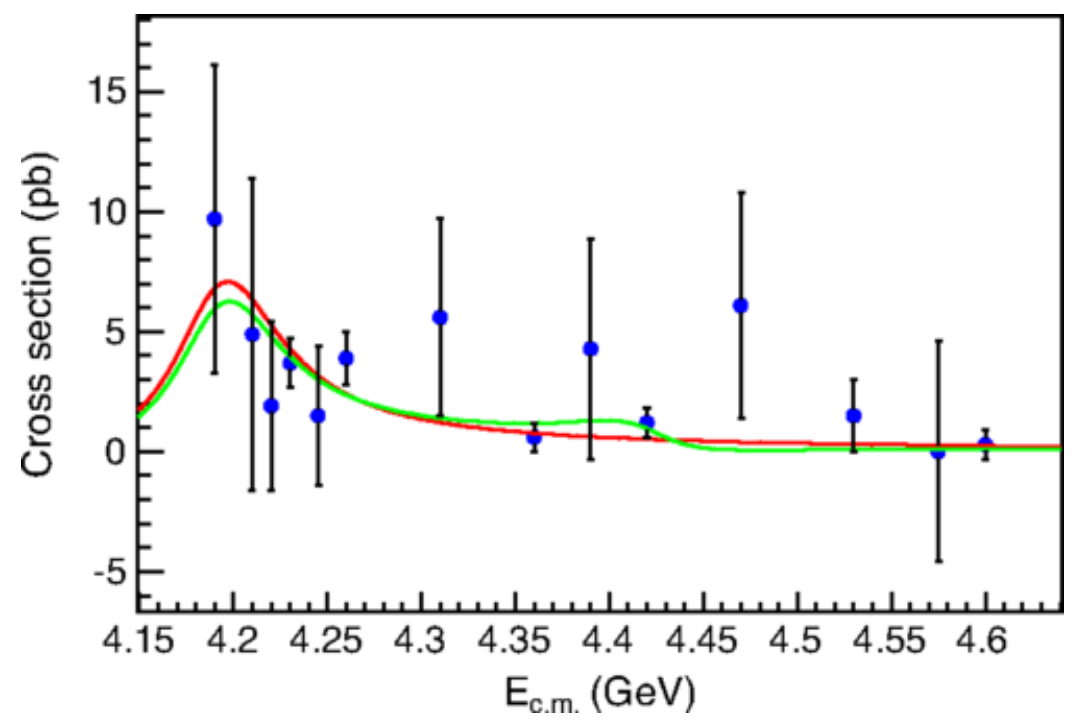

Figure 4: Fit to the Born cross section $\sigma\left(e^{+} e^{-} \rightarrow \eta J / \psi\right)$ with a $\psi(4160)$ resonance (red curve), or a coherent sum of $\psi(4160)$ and $\psi(4415)$ amplitudes (green curve). 
by studying the process $e^{+} e^{-} \rightarrow \pi^{+} \pi^{-} J / \psi$ with $525 p b^{-1}$ data sample at $4.26 \mathrm{GeV}$ [15]. This state can be seen in Fig. 5 (Left). The mass and width of this state are determined to be (3899.0 \pm $3.6 \pm 4.9) \mathrm{MeV} / \mathrm{c}^{2}$ and $(46 \pm 10 \pm 20) \mathrm{MeV} / \mathrm{c}^{2}$, respectively. This state was observed by Belle [9] almost at the same time, and shortly confirmed by a paper using the CLEO-c data [16]. This state can not be a conventional charmonium state, since it carries electric charge. The minimal quark content is a four quark combination.

To reveal the nature of the $Z_{c}(3900)^{ \pm}$state, its neutral partner $Z_{c}(3900)^{0}$ has been searched in the process $e^{+} e^{-} \rightarrow \pi^{0} \pi^{0} J / \psi$ using $2.8 f b^{-1}$ data samples from 4.19 to $4.42 \mathrm{GeV} . Z_{c}(3900)^{0}$ is found with a statistical significance of $10.4 \sigma$, as shown in Fig. 5 (Right). The mass and width are determined to be $(3894.8 \pm 2.3 \pm 2.6) \mathrm{MeV} / \mathrm{c}^{2}$ and $(29.6 \pm 8.2 \pm 7.3) \mathrm{MeV} / \mathrm{c}^{2}$, respectively. The discovery of $Z_{c}(3900)^{0}$ indicates the isospin triplet state $Z_{c}(3900)$ has been established [17].
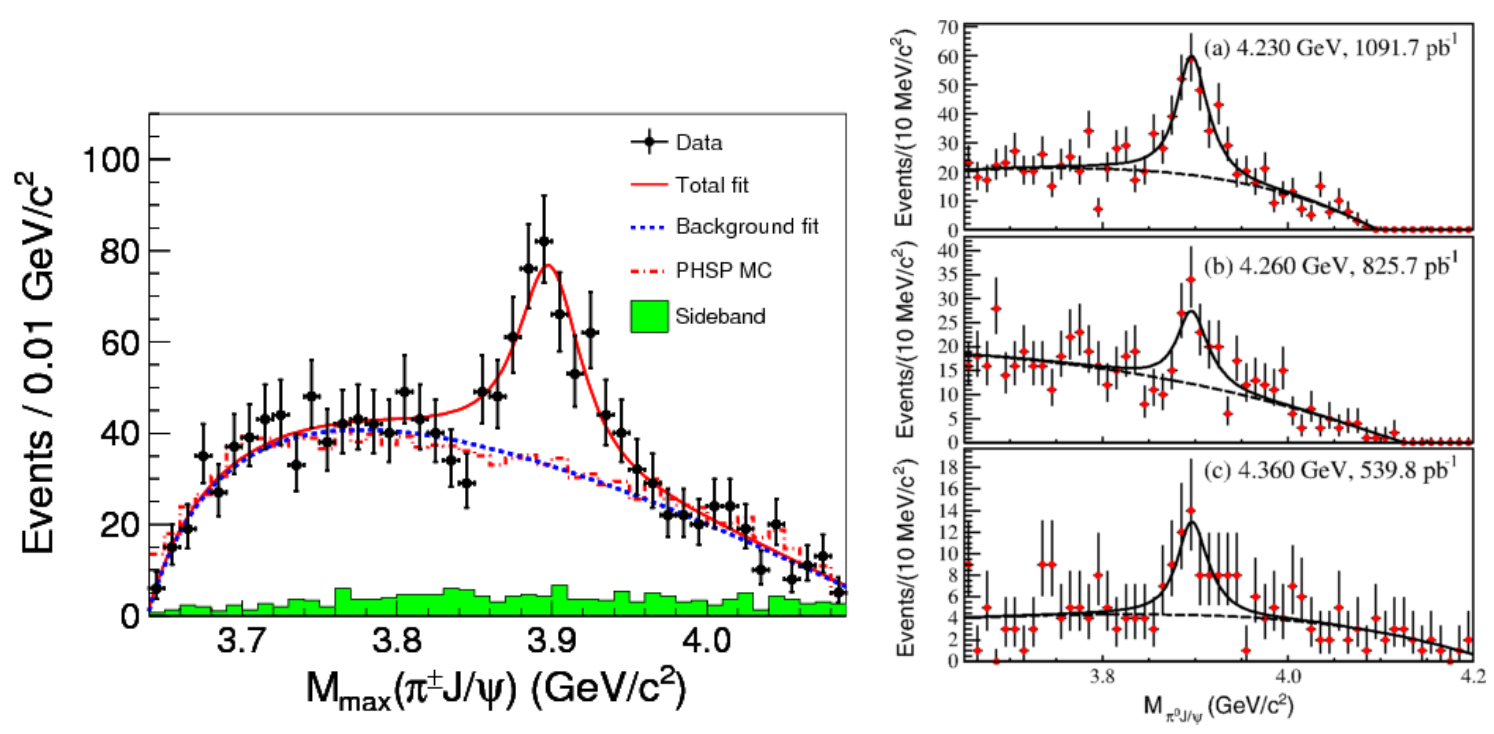

Figure 5: (Left) Fit to the invariant mass of $\pi^{ \pm} J / \psi$. (Right) A simultaneous fit to the $\pi^{0} J / \psi$ mass spectra at three different energy points.

The mass of $Z_{c}(3900)$ is close to the $D \bar{D}^{*}$ threshold, which is suggestive of a virtual $D \bar{D}^{*}$ molecule-like structure. The BESIII experiment performed studies on the process of $e^{+} e^{-} \rightarrow$ $\pi^{ \pm}\left(D \bar{D}^{*}\right)^{\mp}$ with single tag method [18] and double tag method [19]. Using a $525 p b^{-1}$ data sample at $4.26 \mathrm{GeV}$ with the single tag method, an enhancement was observed near the $D \bar{D}^{*}$ mass threshold in the recoil mass of the bachelor pion, as shown in Fig. 6 (Left). This enhancement is named as $Z_{c}(3885)$. The pole mass and width are determined to be $(3883.9 \pm 1.5 \pm 4.2) \mathrm{MeV} / \mathrm{c}^{2}$ and $(24.8 \pm 3.3 \pm 11.0) \mathrm{MeV} / \mathrm{c}^{2}$, respectively. And the quantum number of $Z_{c}(3885)$ is studied by examining the polar angle distribution of the bachelor pion. The signal yield as a function of $\left|\cos \theta_{\pi}\right|$, shown in Fig. 6 (Right), agrees well with the flat expectation for $J^{P}=1^{+}[18]$.

Later, the neutral partner of the $Z_{c}(3885)^{ \pm}$state is observed in the processes $e^{+} e^{-} \rightarrow \pi^{0}\left(D^{+} D^{*-}\right)+$ c.c. and $e^{+} e^{-} \rightarrow \pi^{0}\left(D^{0} \bar{D}^{* 0}\right)+c . c$. [20], which indicates the isospin triplet state $Z_{c}(3885)$ has been established.

Assuming the $Z_{c}$ (3885) structure is due to the $Z_{c}(3900)$, the ratio of partial decay widths is determined to be $\left[\left(\Gamma\left(Z_{c}(3885) \rightarrow D \bar{D}^{*}\right)\right) /\left(\Gamma\left(Z_{c}(3900) \rightarrow \pi J / \psi\right)\right)\right]=6.2 \pm 1.1 \pm 2.7$. This ratio 
is much smaller than the typical values for decays of the conventional charmonium states above the open charm threshold, which suggest the influence of very different dynamics in the $Y(4260)$ $Z_{c}(3900)$ system.
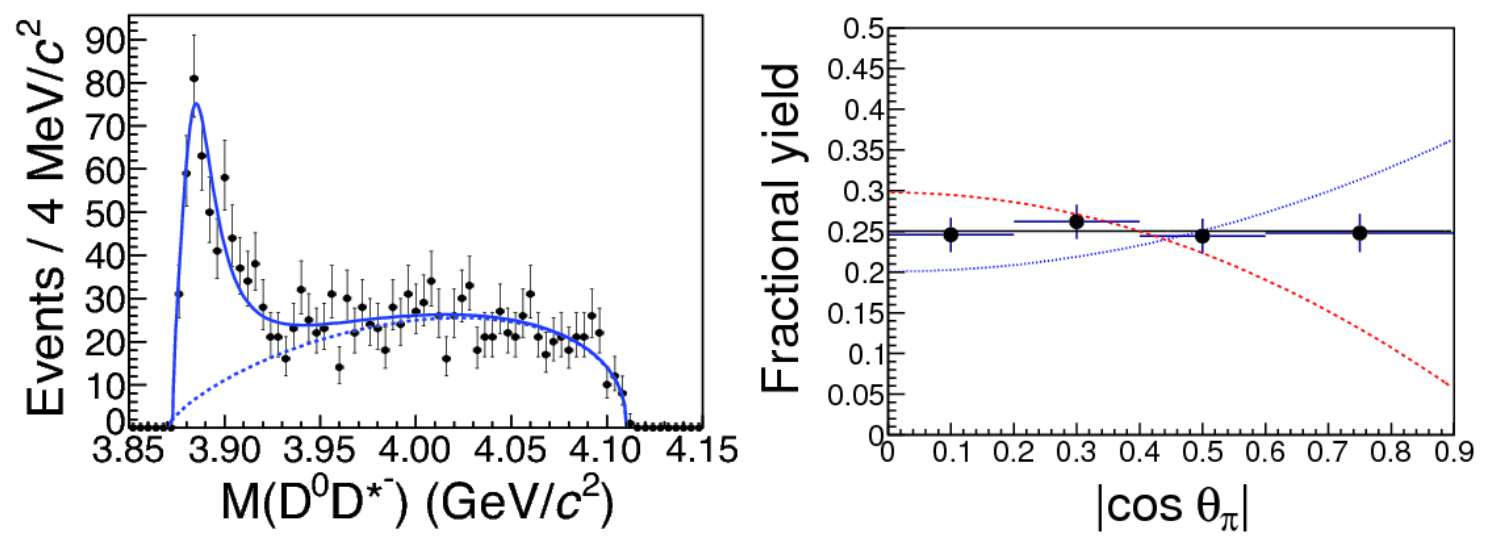

Figure 6: (Left) Fit to the invariant mass of $D^{0} D^{*-}$. (Right) Polar angle distribution of the bachelor pion, in which the solid, dashed, and dotted curves show expectations for $J^{P}=1^{+}, 0^{-}$, and $1^{-}$, respectively.

\subsection{Observations of $Z_{c}(4020)$ and $Z_{c}(4025)$}

In order to search for other $Z_{c}$ states, studies on the process $e^{+} e^{-} \rightarrow \pi^{+} \pi^{-} h_{c}$ was performed at BESIII. In the $M\left(\pi^{ \pm} h_{c}\right)$ spectra, a structure around $4.02 \mathrm{GeV}$ is observed, as shown in Fig. 7 (Left), named as $Z_{c}(4020)$. The mass and width of this state are determined to be $(4022.9 \pm 0.8 \pm 2.7)$ $\mathrm{MeV} / \mathrm{c}^{2}$ and $(7.9 \pm 2.7 \pm 2.6) \mathrm{MeV} / \mathrm{c}^{2}$, respectively. The statistical significance is larger than 8.9 $\sigma[21]$.

Later its isospin partner was observed in the process $e^{+} e^{-} \rightarrow \pi^{0} \pi^{0} h_{c}$. In the $M\left(\pi^{0} h_{c}\right)$ spectra, an obvious peak around $4.02 \mathrm{GeV}$ is seen, as shown in Fig. 7 (Right). A fit on the mass spectra with width fixed to the value from charged channel is applied. The mass is determined to be $(4023.6 \pm$ $2.2 \pm 3.9) \mathrm{MeV} / \mathrm{c}^{2}$, which is consistent with the charged $Z_{c}(4020)$. The statistical significance is larger than $5 \sigma$ [22].
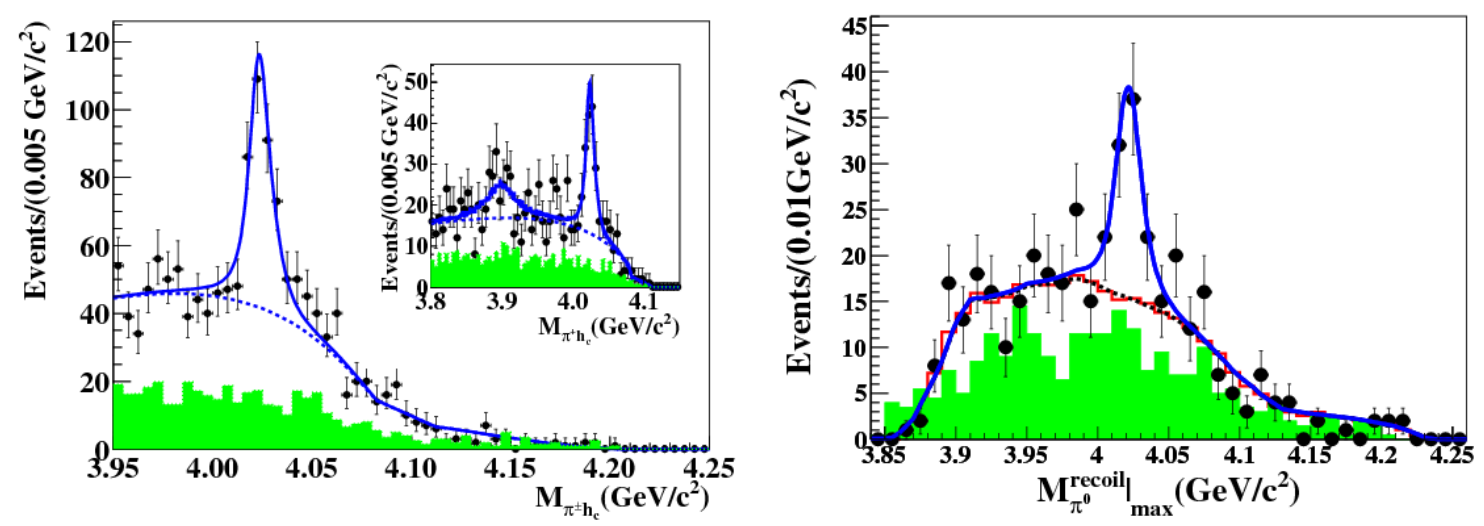

Figure 7: Fit to the invariant mass of $\pi^{ \pm} h_{c}$ (Left) and $\pi^{0} h_{c}$ (Right). 
Since the mass of $Z_{c}(4020)$ is close to the $D^{*} \bar{D}^{*}$ threshold, a search of $Z_{c}$ states in the process of $e^{+} e^{-} \rightarrow \pi^{ \pm}\left(D^{*} \bar{D}^{*}\right)^{\mp}$ was performed. In the recoil mass of the bachelor pion, a structure near the $D^{*} \bar{D}^{*}$ threshold was observed as shown in Fig. 8 (Left), named as $Z_{c}(4025)$. The mass and width of this state are determined to be $(4026.3 \pm 2.6 \pm 2.7) \mathrm{MeV} / \mathrm{c}^{2}$ and $(24.8 \pm 5.6 \pm 7.7) \mathrm{MeV} / \mathrm{c}^{2}$, respectively [23].

Later its neutral partner, $Z_{c}(4025)^{0}$, was observed in the process $e^{+} e^{-} \rightarrow \pi^{0}\left(D^{*} \bar{D}^{*}\right)^{0}$, as shown in Fig. 8 (Right). Assuming a Breit-Wigner line shape, its pole mass and width are determined to be (4025.5 - $\left._{-4.7} \pm 3.1\right) \mathrm{MeV} / \mathrm{c}^{2}$ and $(23.0 \pm 6.0 \pm 1.0) \mathrm{MeV} / \mathrm{c}^{2}$, respectively [24].

Assuming the $Z_{c}(4020)$ and $Z_{c}(4025)$ are the same state, the ratio of the partial decay widths is measured to be $\left[\left(\Gamma\left(Z_{c}(4025) \rightarrow D^{*} \bar{D}^{*}\right)\right) /\left(\Gamma\left(Z_{c}(4020) \rightarrow \pi h_{c}\right)\right)\right]=12 \pm 5$, which is also much smaller than that of the conventional charmonium states above open charm threshold.
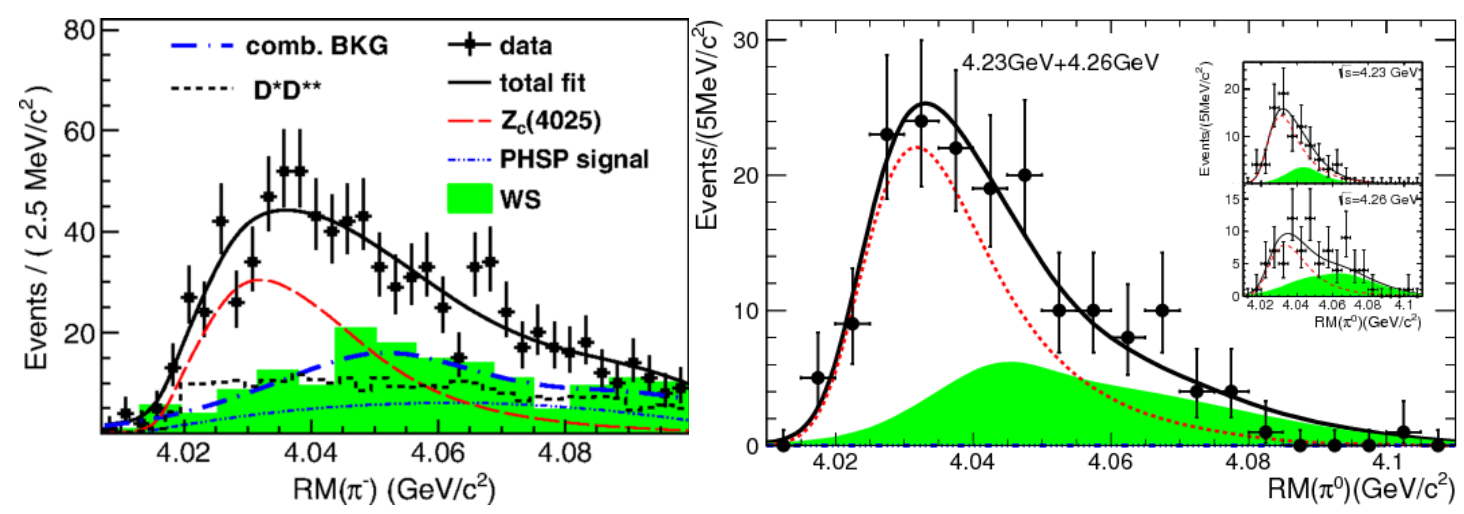

Figure 8: (Left) Fit to the recoil mass of $\pi^{-}$. (Right) Fit to the recoil mass of $\pi^{0}$

\section{Summary}

Based on high luminosity data sets in BESIII, a relatively systematic study on exotic and charmonium-like states has been perform. Radiative and hadronic transitions between higher vector charmonium(-like) states to exotic/conventional charmonium states have also been investigated. Many interesting results have been obtained, which have greatly improved our knowledge about these exotic states. The observation of the charged $Z_{c}$ states may indicate one kind of the exotic states has been observed. However, it is still a big challenge to understand the nature of these exotic states. More data from BESIII experiment is expected to improve the current situation.

\section{References}

[1] H. Fritzsch, M. Gell-Mann and H. Leutwyler, Phys. Lett. B 47, 365 (1973)

[2] V. Bhardwaj et al. [Belle Collaboration], Phys. Rev. Lett. 111, 032001 (2013)

[3] M. Ablikim et al. [BESIII Collaboration], Phys. Rev. Lett. 115, 011803 (2015)

[4] S. K. Choi et al. [Belle Collaboration], Phys. Rev. Lett. 91, 262001 (2003)

[5] M. Ablikim et al. [BESIII Collaboration], Phys. Rev. Lett. 112, 092001 (2014) 
[6] T. Aaltonen et al. [CDF Collaboration], Phys. Rev. Lett. 102, 242002 (2019)

[7] M. Ablikim et al. [BESIII Collaboration], Phys. Rev. D. 91, 032002 (2015)

[8] J. P. Lees et al. [BABAR Collaboration], Phys. Rev. D. 86, 051102 (2012)

[9] Z. Q. Liu et al. [Belle Collaboration], Phys. Rev. Lett. 110, 252002 (2013)

[10] B. Aubert et al. [BABAR Collaboration], Phys. Rev. Lett. 98, 212001 (2007)

[11] X. L. Wang et al. [Belle Collaboration], Phys. Rev. D. 91, 112007 (2015)

[12] M. Ablikim et al. [BESIII Collaboration], Phys. Rev. Lett. 114, 092003 (2015)

[13] M. Ablikim et al. [BESIII Collaboration], Phys. Rev. D. 93, 011102 (2016)

[14] M. Ablikim et al. [BESIII Collaboration], Phys. Rev. D. 94, 032009 (2016)

[15] M. Ablikim et al. [BESIII Collaboration], Phys. Rev. Lett. 110, 252001 (2013)

[16] T. Xiao, S. Bobbs, A. Tomaradze, Kamal K. Seth, Phys. Lett. B 727, 366 (2013)

[17] M. Ablikim et al. [BESIII Collaboration], Phys. Rev. Lett. 115, 112003 (2015)

[18] M. Ablikim et al. [BESIII Collaboration], Phys. Rev. Lett. 112, 022001 (2014)

[19] M. Ablikim et al. [BESIII Collaboration], Phys. Rev. D. 92, 092006 (2015)

[20] M. Ablikim et al. [BESIII Collaboration], Phys. Rev. Lett. 115, 022002 (2015)

[21] M. Ablikim et al. [BESIII Collaboration], Phys. Rev. Lett. 111, 242001 (2013)

[22] M. Ablikim et al. [BESIII Collaboration], Phys. Rev. Lett. 113, 212002 (2014)

[23] M. Ablikim et al. [BESIII Collaboration], Phys. Rev. Lett. 112, 132001 (2014)

[24] M. Ablikim et al. [BESIII Collaboration], arXiv: 1507.02404 